\title{
Near Infrared Transmission through Various Clothing Fabrics
}

\author{
Aamer Saleem, Céline Canal, Lee AJ Davis, Roger J Green and David A Hutchins*
}

School of Engineering, University of Warwick, Coventry CV4 7AL, UK

\begin{abstract}
The transmission characteristics of radiation in the near-infrared range through fabric and similar materials have been investigated, to determine the manner in which the spatial distribution and amplitude levels of the through-transmitted signals are affected by the various physical characteristics of the sample material. A variety of fabric samples have been tested and factors such as material porosity, thickness and moisture content are seen to affect the through-transmission characteristics. Relationships have accordingly been derived to qualify the relative influence of such factors on signal amplitudes and distribution. The work is of interest to near infrared personal security screening applications.
\end{abstract}

Keywords: NIR transmission; Scattering; Fabrics; Porosity

\section{Introduction and Background}

This research formed part of a study aimed at investigating the feasibility of detecting and quantifying various chemicals hidden behind clothing layers in a security screening application [1,2]. In this context, an important variable that had a large effect on the resultant measurement was the transmission characteristics of NIR signals through clothing [3]. It was thus thought important that this was studied in some detail. Of interest was the way in which both the fibre material and the weave pattern affect the transmitted NIR signal, in terms of the total intensity transmitted, the spatial variations in the transmitted beam (i.e. the resultant diffraction pattern) [4] and the effect on transmitted spectra [5].

Experiments were carried out to investigate the way in which scattering took place as a collimated beam of NIR radiation was transmitted through various thicknesses of different types of material. A selection of clothing materials was chosen for study. However, clothing is a complicated case; hence, it was decided to study a different material initially, to try and establish some basic properties, before the more difficult task of understanding transmission through clothing was attempted. Paper was chosen, as it provided a relatively thin and uniform scattering medium [6] compared to fabric samples [7]. In addition, paper is available in known densities and thicknesses as a random mesh. It was thus chosen to provide insight into the transmission characteristics of NIR signals though thin, scattering layers.

\section{Initial Experiments with Paper Layers}

The initial material chosen was 80 gsm paper, 'gsm' being an industry unit for the density of paper in Grams per Square Meter. Each paper sample was placed in the path of an incident $5 \mathrm{~mW}$ NIR laser beam from a laser diode, operating at a wavelength of $850 \mathrm{~nm}$. The beam had a $5 \mathrm{~mm}$ cross-sectional diameter at the sample (full width at half maximum), which was itself perpendicular to the incident laser beam. The through-transmitted energy was recorded using a NIR photodiode, housed in a metal casing with a2 $\mathrm{mm}$ aperture. This was then mounted on a $2 \mathrm{D}$ scanning stage. The scan geometry was set to measure NIR intensity values in the plane perpendicular to the direction of propagation of the NIR beam (i.e. in the $x-y$ plane, across a section parallel with the sample). A schematic diagram and photograph of the experimental arrangement is shown in figure 1. Here, the incident laser diode and the paper samples were held stationary, while crosssectional measurements of transmitted optical intensity were recorded by moving the detector with the $2 \mathrm{D}$ scanning stage shown. Signal enhancement was carried out by modulating the transmitted beam with a $200 \mathrm{~Hz}$ carrier frequency, the latter used as a reference to perform lock-in amplification of the received signal [8]. This allowed detection of through-transmitted signals buried in noise which was many orders of magnitude higher than the recorded signal level, enabling detection of signals across multiple layers of paper. Note that this was important, as very low signal levels would be expected in a real NIR measurement for personal screening purposes. Note that data could also be obtained along the beam axis, if required, by rearrangement of the scanning stages.

In the first experiments, radial spreading of the beam was observed, following transmission through an incremental number of paper sheets. The sheets were held horizontally in a frame. Sheets could be added one at a time, starting with one sheet of paper and going up to forty sheets. The longitudinal displacement of the detector from the sample was kept constant at $6.8 \pm 0.1 \mathrm{~cm}$. The total scan area covered in each case was $80 \mathrm{~mm} \times 80 \mathrm{~mm}$, with a step size of $1 \mathrm{~mm}$. As shown in figure 1 , the scan was controlled by a PC, which controlled the whole

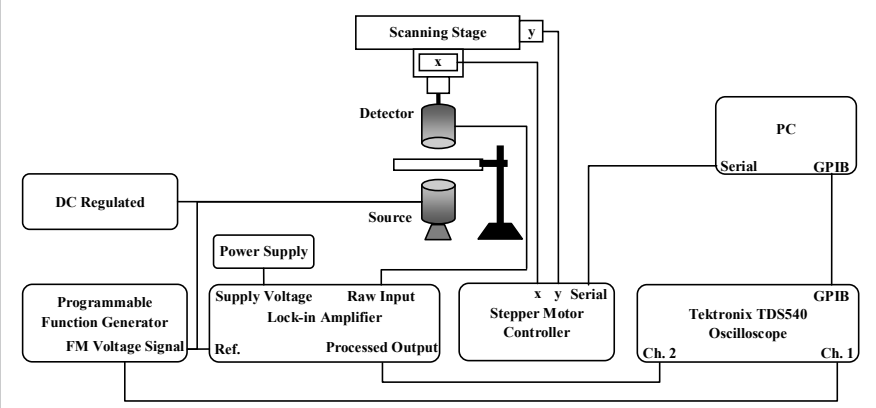

Figure 1: Schematic diagram of the apparatus used to detect throughtransmitted intensity cross-sections in $x-y$ plane, parallel to the test sample. Simple rearrangement of the scanning stages also allowed data to be recorded by scanning the detector along the NIR beam axis.

*Corresponding author: David A Hutchins, School of Engineering, University of Warwick, Coventry CV4 7AL, UK, E-mail: D.A.Hutchins@warwick.ac.uk

Received March 18, 2013; Accepted May 02, 2013; Published May 06, 2013

Citation: Saleem A, Canal C, Davis LAJ, Green RJ, Hutchins DA (2013) Nea Infrared Transmission through Various Clothing Fabrics. J Textile Sci Eng 3: 129. doi:10.4172/2165-8064.1000129

Copyright: ( 2013 Saleem A, et al. This is an open-access article distributed under the terms of the Creative Commons Attribution License, which permits unrestricted use, distribution, and reproduction in any medium, provided the original author and source are credited. 
experiment viaLabVIEW ${ }^{\mathrm{TM}}$ software, with the output from the lock-in being recorded on a digital oscilloscope. The motors were switched off after each scan to a new position before data were collected, to reduce noise from the motors. As in this and other experiments to be described below, the data is for a single measurement; no repetition or data averaging was performed.

Cross-sectional intensity patterns were recorded, both with no sample present and with sheets of paper being inserted into the beam, one on top of the other. The results are shown in figures $2 a$ and $2 b$ for the beam only and with a single layer of paper present respectively. It can be observed that there was considerable broadening of the beam when the sheet of paper was placed between the source and the detector, as would be expected from scattering. Further, the highest intensity readings were concentrated in the centre of the beam (coincident with the axis of the incident beam), with rapidly decreasing intensities radially outwards from the centre.

When additional layers were added, the overall transmission intensity was seen to reduce with increasing number of paper sheets, as would be expected. However, the intensity distribution pattern remained essentially the same. To illustrate this, the Full Width at Half Maximum (FWHM) values [9] of the through-transmitted intensity profiles were measured for cross-sectional line scans. This was done for multiple paper layers, at a distance of $z=1 \mathrm{~mm}$ from the top paper surface (Figure 1). Normalized intensity curves for the forty scans are shown in Figure 3a, with the corresponding FWHM values plotted in Figure 3 b. As can be seen, the FWHM values clearly show an approximately linear upward trend save for a few anomalous readings attributable to experimental errors, with an almost $40 \%$ increase in magnitude as the number of scatterers is increased from 1 to 40 . This shows that the FWHM value of the through-transmitted intensity profile increased approximately linearly with the thickness of the scattering medium in terms of the total thickness of papers with different scattering media detailed in the following sections.

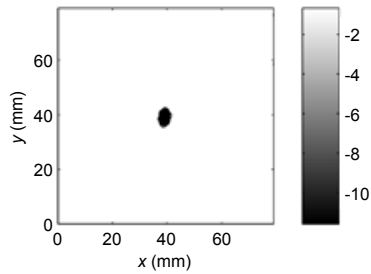

(a)

Figure 2: Cross-sectional intensity scans recorded in the $x-y$ plane for (a) no sample and (b) for a single layer of paper as the scatterer.
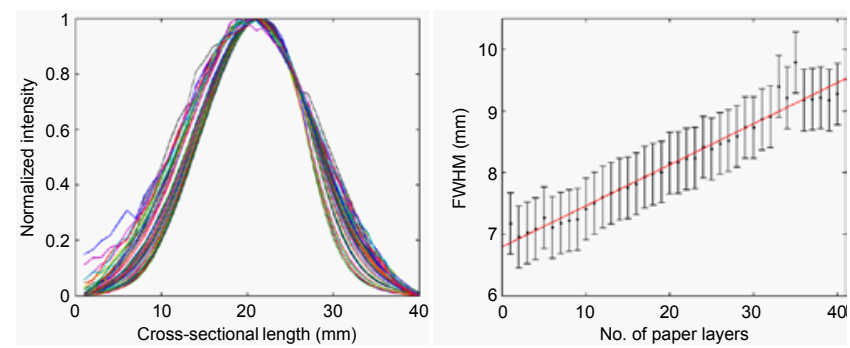

(b)

Figure 3: Results of cross-sectional line scans across 1-40 sheets of paper; (a) Normalized intensity curves; (b) FWHM values for the curves in (a).

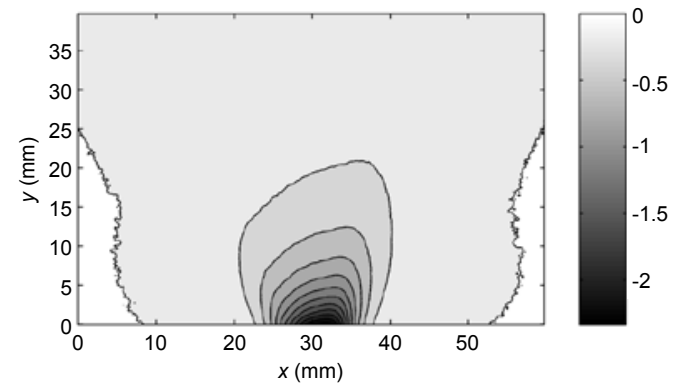

Figure 4: Intensity scan in a plane collinear with the direction of propagation of the beam, with the addition of a single layer of paper between the incident beam and the scanned detector.

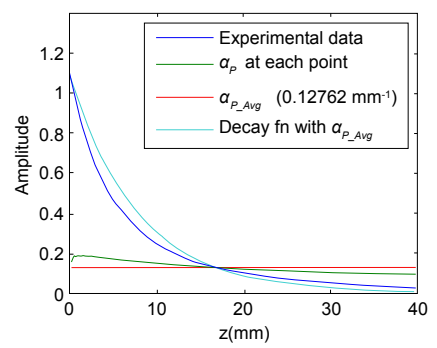

(a)

Fure 5: Axial changes in through-transmitted optical intensity values as the photodiode detector was scanned along the z-axis, away from the scattering paper sample. (a) Characteristic plots for paper, and (b) $\alpha_{p} A v g$ for 1-5 layers of paper, where a linear relationship with the number of paper layers was observed.

Figure 4 shows the spatial variations in optical intensity recorded for a single layer of paper in the $x-z$ plane, namely in a plane collinear with the transmission axis, using the apparatus arrangement shown in figure 1 .

The through-transmitted intensity pattern for paper showed a marked lateral broadening, as expected from figures 2 and 3.It should be noted that, within the resolution of the photodiode detector, there was no detectable divergence in the beam up to the limit of the scanned distance (approximately $35 \mathrm{~cm}$ from the source) in the absence of a sample. It was also observed that, while overall intensity values decreased with increasing numbers of paper layers, the overall pattern of the through-transmitted intensity distribution with axial distance remained similar to that shown in figure 4 .

The decay in optical intensity as the transmitted signal propagated away from the far side of a paper sample was also studied. Intensity readings were recorded along the main beam axis (i.e. along the $z$-axis) for increasing number of paper layers. The intensity readings recorded with two sheets of paper are shown in Figure 5a, where the decay appears to be approximately exponential. A simple relationship that describes the drop in axial intensity with distance $z$ can be written as [10]:

$$
I=I_{0} e^{-\alpha p z}
$$

Here, the instantaneous intensity $I$ is linked to initial intensity $I_{0}$ via $\alpha_{p}$ (the propagation attenuation coefficient) and $z$ (the distance along the $z$-axis). The value of $\alpha_{P}$ can thus be calculated for each point along the $z$-axis using:

$$
\alpha_{p}=-\frac{1}{z} \ln \frac{I}{I_{0}}
$$


All calculated $\alpha_{p}$ values plotted in Figure 5a were then averaged to obtain $\alpha_{p \_} A v g$ as shown. Using this average value of $\alpha_{p}$, the exponential decay curve based on (1) was obtained and plotted as shown. As seen, this curve closely follows the trajectory of the experimental data, thus validating the original approximation to such an exponential function.

The above procedure was used to obtain values of $\alpha_{p}$ across 1-5 layers of paper. These are plotted against respective number of layers in Figure 5b. The plot exhibits an approximately linear characteristic, with $\alpha_{p \_} A v g$ decreasing as the number of paper sheets is increased. This indicates that for a single sheet of paper, intensity values start from a relatively high initial level and decrease rapidly along the z-axis with a correspondingly high $\alpha_{p-} A v g$. As the number of layers (i.e. thickness of the scattering medium) is increased, the initial intensity readings decrease substantially, and the measured intensity then decays more gradually, as characterised by decreasing values of $\alpha_{p \_} A v g$.

In summary, these measurements indicated that the likely effect of scattering within media such as paper is that beam cross-sections increase on transmission through more layers, with the peak intensity decreasing. Once these beams propagate away from the sample on the far side, the decay in axial intensity is exponential, with a characteristic value of $\alpha_{p \_} A v g$ the value of which decreases with the number of paper

(a)
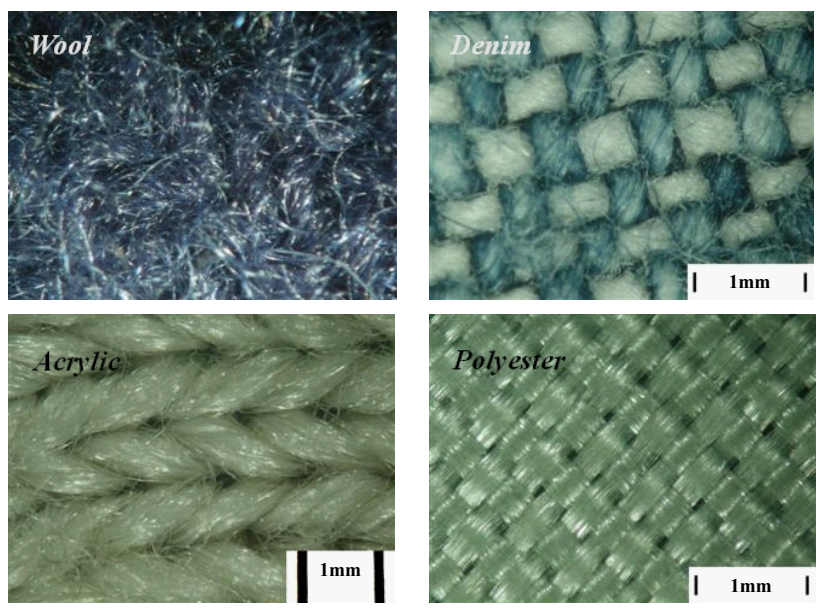

(b)
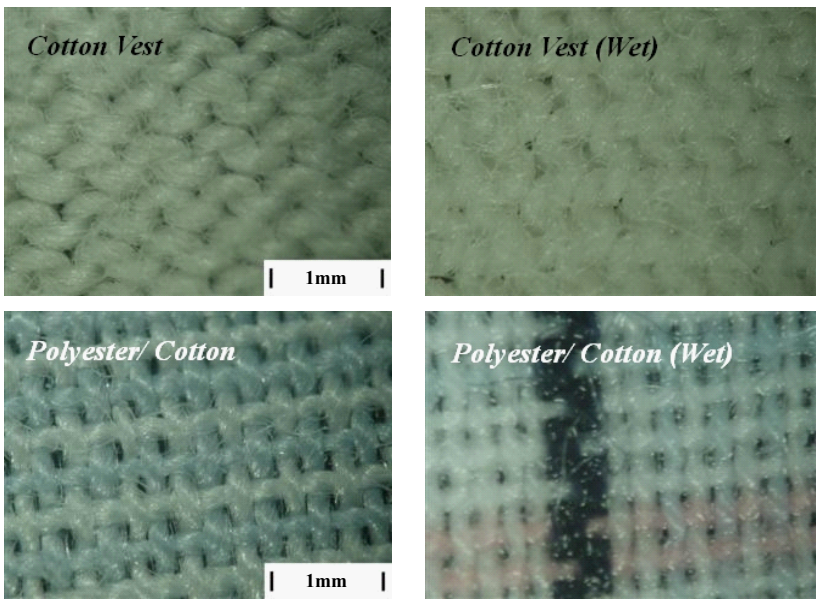

Figure 6: Optical microscope photographs of (a) four of the fabric samples investigated, and (b) some fabrics both in the dry state and when moistened with water. layers/thickness of the sample. The beam cross-section at FWHM appears to depend linearly on the thickness of the sample in such cases.

\section{NIR Scattering in Fabric Samples}

The above has demonstrated the effects likely to be seen in a random, uniform scattering sample, in terms of the effect on the through-transmitted beam. One of the primary concerns in the context of developing a practical personal screening/imaging system is the way in which incident radiation interacts with the clothing materials worn by the subjects in a personal screening test [11]. The weaves found in typical clothing layers are likely to have through-transmission characteristics in the NIR wavelength range that contain aspects of both of the main features mentioned above in section 2 , in that they would be likely to introduce a degree of scattering (due to fibres) which would cause beam spreading. In addition, however, the various differences in fabric material and weave would lead to differences in porosity, which would also affect overall transmission levels. It was thus thought important to study a range of different fabric materials.

In order to gain an appreciation of the different phenomena that dictate the behaviour of various clothing materials, a range of different fabric samples was used to carry out comparative tests. Photographs of some of these samples taken under an optical microscope are shown in figures $6 \mathrm{a}$ and $6 \mathrm{~b}$, where the latter includes two cotton samples in both dry and wet states. Note that wetting seems to have caused the fibres to swell, decreasing the porosity of the samples. The samples include a range of porosities, yarn characteristics and weave patterns, including both man-made and natural materials. Different colours were also present.

All the samples were exposed in turn to an NIR laser beam at $850 \mathrm{~nm}$ using the same arrangement as shown in figure 1, and point measurements were made to record intensity levels with the photodiode detector axially aligned with the laser source. Measurements were taken across increasing number of layers of the fabric samples until no further signal could be detected. The results, shown in figure 7 , illustrate the general overall decrease in signal levels with increasing number of layers. Note that the rate of intensity decrease varies greatly between samples-denim, for example, caused complete attenuation of throughtransmitted energy within 2-3 layers, whereas cotton and polyester samples provided measurable signal levels even through 10-12 layers.

Further tests were undertaken to test whether the colour of a particular fabric affected through-transmitted energy level, noting that the $850 \mathrm{~nm}$ wavelength used for the NIR signal was not within the visible spectrum [12]. Five samples of a white cotton fabric were

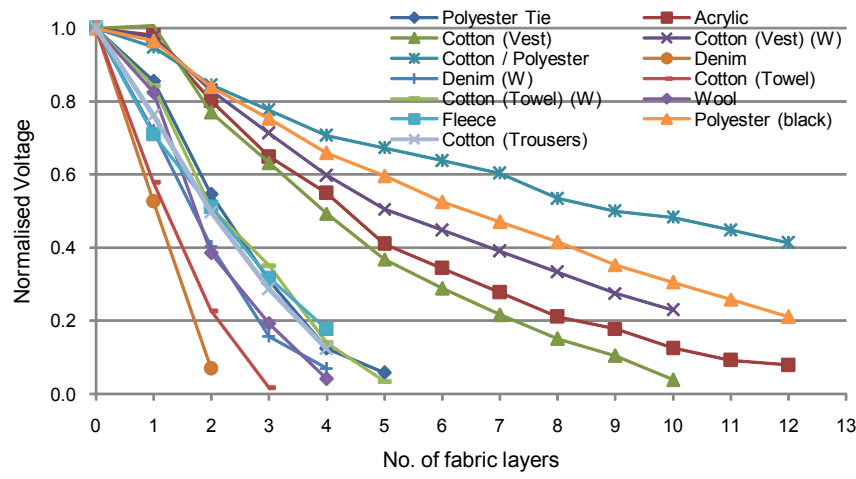

Figure 7: Point measurements showing drop in NIR intensity across multiple fabric layers. (W) signifies wet samples. 
dyed red, green, blue, yellow and black, and a further two samples of a polyester fabric were obtained in white and black colours. The normalized through-transmitted signal levels recorded across these samples are shown in figure 8. While these results show some variation between the different colours, this is deemed insignificant compared with the differences amongst the different fabric types, and can be attributed to the different chemical compositions of the relevant dyes affecting the through-transmitted signal levels differently [13].

The data plotted in figures 7 and 8 also include readings for wet samples (marked as 'W'). Cotton was chosen as an initial example, to illustrate the likely effect of water adsorption and fibre swelling on the through-transmitted NIR signal. As seen, wet cotton samples provided higher through-transmission levels than the same samples in the dry state. This effect was further investigated by testing a range of fabric materials, each containing varying levels of moisture. Each sample was saturated with water and allowed to dry through evaporation. Its weight, measured at regular intervals with a microbalance, was used as an indicator of moisture content, and recorded against the throughtransmitted signal level. The results are shown in figure 9. It can be seen that increasing moisture content resulted in an increase in the recorded through-transmitted signal. This higher transmission in the presence of water is thought to be the result of water acting as an index matching layer [14] - the difference between the refractive indices of air [15] and water is smaller than the difference between the indices of air and fabrics [16] allowing reduced refractive index mismatch between air and the sample. This reduces the amount of scattering, resulting in more signal energy emerging on the other side of the sample. Additionally, as the moisture content increases, the water progressively fills in the pores in the fabric, which would render the structure more optically homogeneous with associated higher through-transmitted signal levels.

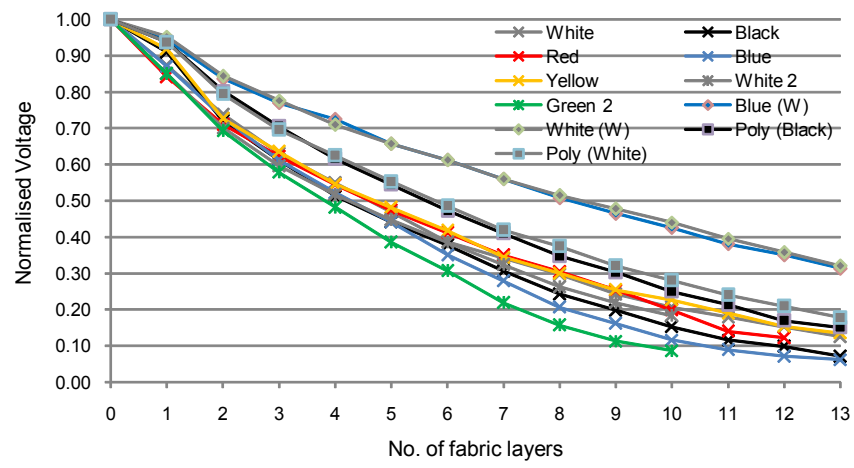

Figure 8: Signal variation across various dyed fabric samples of cotton (six colors) and polyester (black and white only). (W) signifies wet samples.

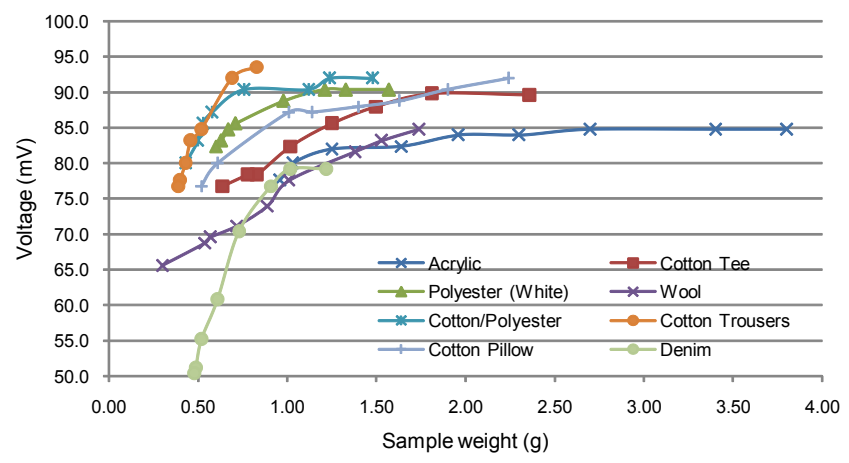

Figure 9: Effect of moisture on through-transmitted signal levels.

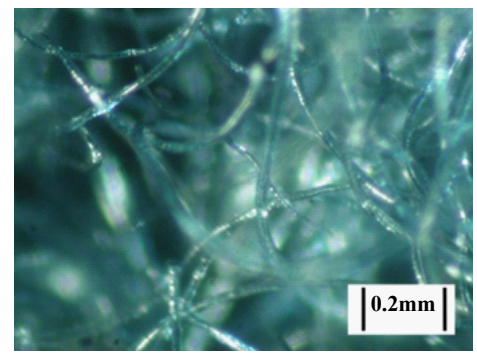

Figure 10: Microscopic image of the wool material, showing a highly porous structure.

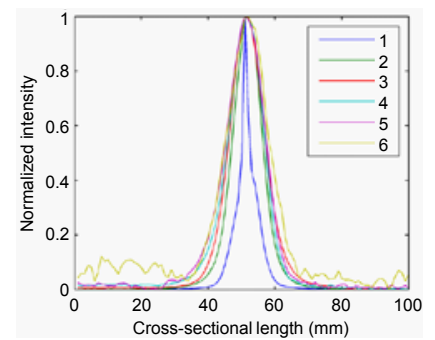

(a)

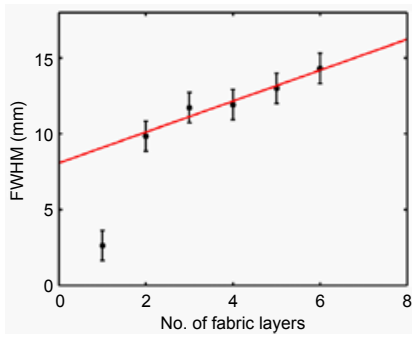

Figure 11: Measurements with a woollen scarf fabric. (a) Normalized crosssectional line scan data for 1-6 layers; (b) FWHM values measured for the line scan curves shown in (a), with the 1st value excluded from the linear fit.

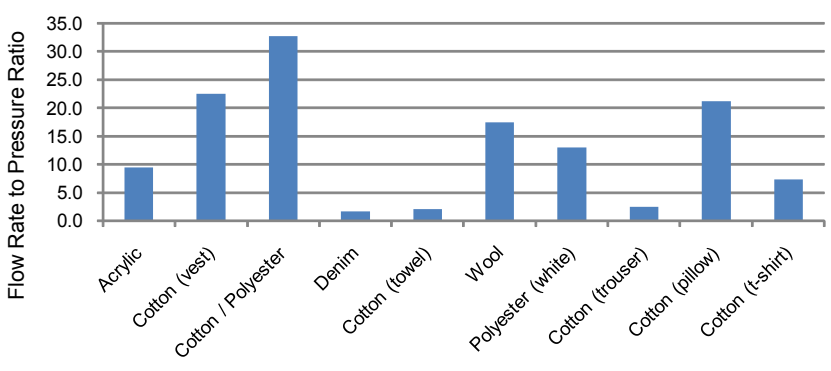

Fabric

Figure 12: Variation in porosity with different fabric samples.

In order to clarify the results obtained with wool, the structure of the sample was examined in optical microscope, and was seen to be highly chaotic as shown in the photograph in figure 10. This can thus be considered a possible limiting case for scattering within fabric samples. Using the scanning system of figure 1 , line scans were obtained across incremental layers of the woollen scarf of up to 6 layers. The results of cross-sectional line scans, and the resulting FWHM values, are plotted for the respective number of layers in figure $11 \mathrm{a}$ and $11 \mathrm{~b}$ respectively. As expected, the FWHM values exhibit an approximately linear dependence with increasing number of layers (or thickness of the fabric medium) for two or more layers. Note however the low FWHM value when only one layer was tested. It would thus appear that, for this particular highly-porous material, the behaviour deviates from that of the much less porous paper sample, for example, for small thicknesses of material; once thicker overall transmission thicknesses are present, a similar behaviour is observed (see comparison of figure $3 \mathrm{~b}$ and figure $11 b)$.

The studies above have shown that the transmission characteristics are a complicated function of the weave pattern and the material 
used for the fibres. To try and establish a clearer link with porosity, a set of experiments was performed on fabric samples to measure their porosity [17], and link this to overall NIR transmission levels. Porosity measurement involved exposing the fabric samples, held between two plates in a mass flow meter; to a stream of air at a pre-determined flow rate $F$. The resulting differential pressure $P$ across the sample was then measured using a Sensirion SDP1000-R low-range differential pressure transducer attached to the mass flow meter. The high pressure input was provided from a compressed air source via a control unit used to adjust the air flow rate as desired, while the low pressure output was connected to a vent with a long stretch of pipe. PressureP was deemed to be inversely proportional to the porosity of the sample at a given flow rateF.The voltage readings provided by the differential pressure transducer were converted to pressure $P$ using the following relationship as per manufacturer's guidelines [18]:

$$
P=35.55556 \times(V-0.25)^{2}
$$

where $V$ denotes measured output voltage in volts, and $P$ is the pressure in pascals. The measure of porosity (Por) was then taken as the ratio of flow rate $F$, recorded in $\mathrm{ml} / \mathrm{min}$, to pressure $P$ :

$$
\text { Por }=F / P
$$

A range of readings was taken with each fabric sample at different flow rates, and averaged to find the final estimate of porosity. The results for all fabric samples are shown in figure 12 .

When these measurements are correlated with the throughtransmission results shown in figures 7 and 8 , it is seen that, in general, higher porosity values provide higher through-transmitted signals and vice versa. For example, the three samples with the lowest porosity estimates, i.e., denim, cotton (towel) and cotton (trousers), are seen to have the lowest transmission values, while the cotton/polyester sample with the highest porosity estimate gives highest transmission readings as well. An interesting exception is wool, which provides the third lowest transmission values (lower than the cotton (trousers) sample), although its porosity estimate is relatively high. This behaviour is deemed to be the result of the unusually chaotic surface texture of wool as seen in figure 10, which scatters more NIR energy than other materials with similar porosity values.

As a final check on the total amount of light transmitted by these fabrics, an integrating sphere was used to collect the throughtransmitted signal [19]. Such instruments collect all the light into an aperture, which then passes into a blackened spherical cavity where the integrated total light intensity is measured. Intensity readings across one and two layers of different fabric samples were recorded using Ocean Optics FOIS-1 integrating sphere connected via an optical fibre cable to an HR4000 Ocean Optics NIR Spectrometer. Note that for these readings, the fabric sample was pressed against the aperture of the integrating sphere, as shown in figure 13.

The intensity readings recorded across four representative fabric samples are shown in figure 14. In contrast to previous measurements described above, the level of total through-transmitted intensity for wool was higher than for cotton. This might be expected from the higher porosity of wool, and also fits with the lack of excessive beam broadening seen for a single layer of wool in figure 11a. Note, however, that acrylic (with a lower porosity than that of wool) still exhibited a greater degree of NIR transmission. This could be the result of greater backscatter from the surface of wool caused by the significantly higher proportion of randomly-distributed scatterers in the path of the impinging NIR radiation. Similarly, greater backscatter could explain

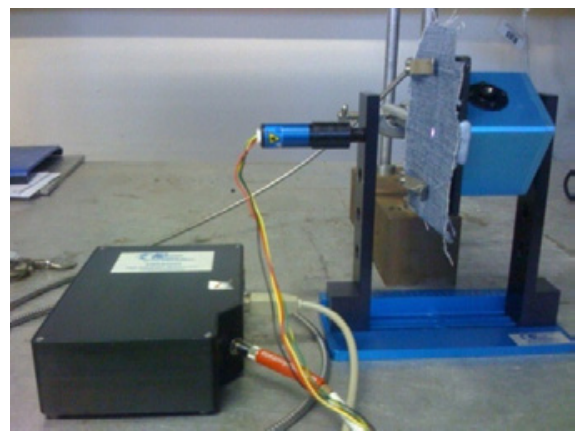

Figure 13: Experiments to measure total transmitted intensity with integrating sphere.

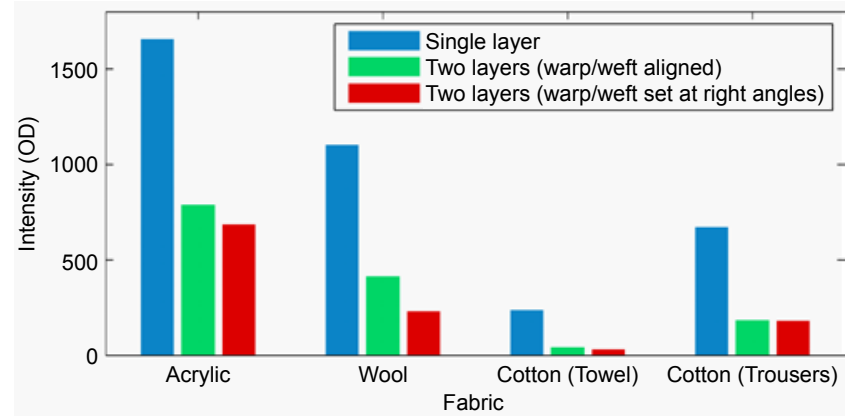

Figure 14: Intensity readings recorded across fabric samples with integrating sphere.

the slightly lower intensity readings recorded with two layers when the warp/weft patterns are set at right angles compared with when they are aligned.

The above results indicate that the magnitude of throughtransmitted radiation depends not only on the overall porosity of the sample, but also on the size and spatial distribution of the pores.

\section{Practical Application}

One of the applications of near infrared transmission through clothing is security screening-the ability to detect the presence of objects hidden behind clothing layers. The way in which scattering occurs will limit the resolution of any imaging that takes place. Hence, an understanding of the processes involved is of importance. As an illustration of the technique, figure $15 \mathrm{a}$ shows an experiment where the clothing material is illuminated by broad-spectrum NIR energy from halogen lamps. Hidden behind this fabric layer is an acylic container filled with a chemical. The aim is to transmit signals through the fabric, and form an image from NIR energy backscattered from the container and its content. A collimating lens collects this light over a narrow angle, and this is then imaged using a Basler Scout 2 video camera sensitive to NIR wavelengths (in this case up to $1.6 \mu \mathrm{m}$ in wavelength). Note also that NIR spectroscopy can also be used if required for chemical identification

Figure 15b shows a typical image that can be obtained. Although the front surface of the fabric reflects some light, it is possible to see the outline of the rectangular container through the clothing material, in this case a cotton shirt with a checked pattern. Note that this pattern does not show up on the image, as the dyes used have only a small effect on the through-transmitted NIR signal amplitude. In this case, 


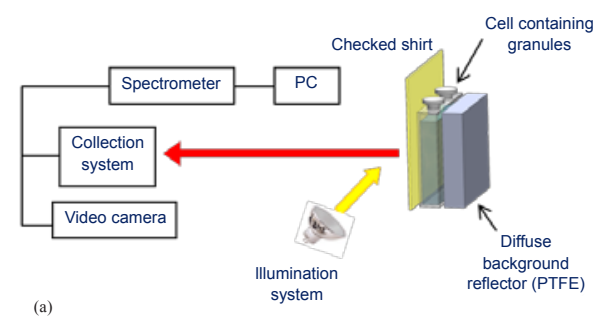

Figure 15: (a) apparatus for investigating through-clothing imaging using NIR signals. The acrylic cell can contain powders or liquids. (b) Image of the cell through the cotton shirt material, showing the outline of the cell. This outline is blurred by the scattering phenomena outlined in the above experiments.

being thin cotton, the outline of the object is only affected slightly by the scattering in the fabric. However, as detailed above, the type of fabric, and the number of layers, both will affect the amount of light transmitted, and the amount of scatter, which will in turn affect the quality of any image obtained.

\section{Conclusions}

Based on the above observations, it may be concluded that the dominant factors in the transmission and scattering of NIR energy at $850 \mathrm{~nm}$ across typical clothing fabrics are the porosity of the fabrics and the spatial distribution of pores. While the overall porosity may determine the total amount of energy that is transmitted across the clothing barrier, the intensity distribution across the transmitted volume is determined by the geometric layout of the fibres, and hence that of the pores. Additionally, the chaotic surface texture of fabrics such as wool may impose further limitations on the amount of transmitted energy notwithstanding the overall porosity. Therefore, dry fabrics may be expected to transmit higher intensities with increasing pore size and more uniform surface texture. Furthermore, introduction of moisture in the fabrics tends to increase transmission levels by reducing the refractive index mismatch at the boundary between air and fabric, and thus reducing the optical impedance of the fabric.

It was observed that the dye used on many fabric samples has only a minor effect on the amount of transmitted NIR energy at the wavelength used.

The application of interest is the imaging of objects behind clothing layers, and an example was included, showing that this is possible. It is evident, however, that the amount of NIR energy transmitted through the fabric, and its spatial distribution, will depend on the type of fabric and its thickness, and hence the research is likely to be valuable as an indication of the possible range of application of the technique.

\section{References}

1. Canal CM, Saleem A, Green RJ, Hutchins DA (2011) Remote identification of chemicals concealed behind clothing using near infrared spectroscopy. Anal Methods 3: 84-91.

2. Saleem A, Canal C, Hutchins DA, Davis LAJ, Green RJ (2011) Techniques for quantifying chemicals concealed behind clothing using near infrared spectroscopy. Anal Methods 3: 2298-2306.

3. Zhou Y, Xu Hr, Ying Yb (2008) NIR analysis of textile natural raw material Spectroscopy and Spectral Analysis 28: 2804-2807.
4. Millan MS, Escofet J, Rallo M (2010) Unsupervised defect segmentation of patterned materials under NIR illumination. $17^{\text {th }}$ Reunion Iberoamericana de Optica, RIAO and $10^{\text {th }}$ Encuentro de Optica, Laseres y Aplicaciones, OPTILAS, Lima, Peru.

5. Wu G, He Y (2007) Identification of fine wool and cashmere by Vis/NIR spectroscopy technology. International Symposium on Photoelectronic Detection and Imaging: Related Technologies and Applications, USA.

6. Havermans J, Aziz HA, Penders N (2005) NIR as a tool for the identification of paper and inks in conservation research. Restaurator 26: 172-180.

7. Yuan HF, Chang RX, Tian LL, Song CF, Yuan XQ, et al. (2010) Study of nondestructive and fast identification of fabric fibers using near infrared spectroscopy Guang Pu Xue Yu Guang Pu Fen Xi/Spectroscopy and Spectral Analysis 30: 1229-1233.

8. Corluka V Filic M, Valter Z (2005) Lock-in amplifier in NIR reflectance measurement system in Annals of DAAM. Proceedings of the 16th International DAAM Symposium "Intelligent Manufacturing and Automation: Focus on Young Researches and Scientists", Vienna, Austria.

9. Markevich N, Gertner I (1989) Comparison among methods for calculating FWHM. Nuclear Instruments \& amp; Methods in Physics Research.

10. Tagesen S, Winkler G (1992) Troubles, traps and tricks in fitting exponential decay data. International Symposium on Nuclear Data Evaluation Methodology, Singapore, Singapore.

11. Bjarnason JE, Chan TLJ, Lee AWM, Celis MA Brown ER (2004) Millimeterwave, terahertz, and mid-infrared transmission through common clothing Applied Physics Letters 85: 519-521.

12. Huang W, Wang G, Xu X, Yu T, Yang Z (2010) Forensic inspection of document using visible and near-infrared spectral imaging. Optoelectronic Imaging and Multimedia Technology, USA.

13. Tavanai $H$, Hamadani AZ, Valizadeh $M$ (2003) Colour yield in two phase wet fixation dyeing of cotton cloth with reactive dyes as a function of time, temperature and alkali concentration. Iranian Polymer Journal 12: 459-475.

14. Kronig R, Blaisse B, Sande JJvd (1950) Optical impedance and surface coating. Applied Scientific Research Section B 1: 63-76.

15. Hecht E (2002) Optics (4th edn.). Pearson Addison Wesley, Reading, UK.

16. Barakat N, El-Hennawi HA (1971) Interferometric Studies of Fibers. Textile Research Journal 41: 391-396.

17. Ogulata RT, Mavruz S (2010) Investigation of porosity and air permeability values of plain knitted fabrics. Fibres \&amp; Textiles in Eastern Europe 18 $71-75$

18. SDP1000/SDP2000 (2008) Low Range Differential Pressure Sensor for Air and Non-Aggressive Gases, Datasheet.

19. Ojala KT, Koski E, Lampinen MJ (1992) Reflection and transmission measurements with an integrating sphere and Fourier-transform infrared spectrometer. Applied Optics 31: 4582-4589. 